in vivo $35: 461-465(2021)$

doi:10.21873/invivo.12279

\title{
Radiation to the Primary Tumor in Metastatic Anaplastic Thyroid Cancer
}

\author{
TERESA AUGUSTIN ${ }^{1}$, DMYTRO OLIINYK ${ }^{1}$, JOSEFINE RAUCH ${ }^{1}$, VIKTORIA FLORENTINE KOEHLER ${ }^{2}$, \\ CHRISTINE SPITZWEG ${ }^{2,3}$, CLAUS BELKA ${ }^{1,3}$ and LUKAS KÄSMANN ${ }^{1,3}$ \\ ${ }^{1}$ Department of Radiation Oncology, University Hospital, LMU Munich, Munich, Germany; \\ ${ }^{2}$ Department of Internal Medicine IV, University Hospital, LMU Munich, Munich, Germany; \\ ${ }^{3}$ German Cancer Consortium (DKTK), Partner Site Munich, Munich, Germany
}

\begin{abstract}
Background/Aim: Metastatic anaplastic thyroid cancer is associated with a dismal prognosis. We evaluated outcome and prognostic factors in patients receiving radiation to the primary tumor in metastatic anaplastic thyroid cancer (ATC). Patients and Methods: All consecutive patients with metastatic ATC $(n=20)$ undergoing irradiation between 2009 and 2019 for anaplastic thyroid cancer were investigated. Results: Median survival time and median progression-free survival were 2 (range=1-22) and 2 (1-20) months. In univariate analyses, surgery, concurrent or sequential chemotherapy and higher radiation dose escalation (>39 Gy) were correlated with longer overall survival ( $p=0.005$, $p=0.018$ and $p=0.038)$, respectively. Karnofsky performance status $>70 \%$ showed a trend of longer survival time ( $p=0.062)$. Limited metastatic disease, surgery and concurrent/sequential chemotherapy are correlated with longer progression-free survival times $(p=0.043, p=0.024$ and $p=0.039)$, respectively. Conclusion: Radiation to the primary tumor in metastatic anaplastic thyroid cancer is safe and offers durable local control. Treatment intensification including concurrent or sequential chemotherapy and radiation dose escalation were associated with longer survival rates and should be considered in selected patients with metastatic disease.
\end{abstract}

Anaplastic thyroid cancer (ATC) is an orphan disease with a dismal prognosis (1-6). Median survival times range between

This article is freely accessible online.

Correspondence to: Lukas Käsmann, Department of Radiotherapy and Radiation Oncology, Ludwig-Maximilians-Universität, Marchioninistraße 15, 81377 München, Germany. Tel: +49 89440073770, Fax: +4989440076770, e-mail: lukas.kaesmann@ med.uni-muenchen.de

Key Words: ATC, anaplastic thyroid cancer, metastatic, irradiation, survival.
3 and 16 months depending on Union for International Cancer Control tumor-node-metastasis (UICC TNM) stage (7-9). National and international guidelines recommend that ATC patients with stage IVA/B should be considered for radical surgery if negative pathological margins (R0/R1) can be achieved and should be avoided if there is a high chance of gross residual disease (R2) (2-5).

However, the majority of ATC patients are considered to be inoperable due to locally advanced disease and comorbidities. So far, no standard treatment regimen has been successfully established in stage $\operatorname{IVC}(4,5)$.

Local radiotherapy (RT) should always be considered as part of the initial multimodal therapy approach to improve overall survival (OS) $(6,9-11)$ and provide local and symptom control in order to maintain quality of life. However, it is a matter of debate if local RT is still recommended in metastatic disease where aggressive systemic therapy has to be weighed against benefits from local control and limited systemic approaches.

The aim of the present study was to evaluate outcome and prognostic factors in patients receiving radiation to primary tumor of metastatic anaplastic thyroid cancer.

\section{Patients and Methods}

Study population. The medical charts of 20 consecutive patients with metastatic anaplastic thyroid cancer treated with irradiation to primary tumor between 2009 and 2019 were reviewed. Patient and treatment characteristics are summarized in Table I. The Institutional Research Ethical Review Board approved the study (approval number: 19-885).

Diagnostics and treatment. ATC was histologically confirmed in all patients and diagnosed as stage IVC according to the revised 8th edition of the UICC TNM classification (7). A lobectomy was performed in two (10\%) patients and three $(15 \%)$ patients had received a total thyroidectomy. Chemotherapy was administered in 9 (45\%) patients, of which $5(25 \%)$ patients received concurrent chemotherapy with carboplatin AUC 2 with Paclitaxel $50 \mathrm{mg} / \mathrm{m}^{2}$ weekly, which has been the standard at our Institution in the recent years, and 3 patients (15\%) received concurrent chemotherapy with 
doxorubicin (10 or $20 \mathrm{mg} / \mathrm{m} 2)$ weekly. Sequential chemotherapy (doxorubicin/cisplatin) was administered in one (5\%) patient before radiation. Three-dimensional conformal radiotherapy (3D-CRT) technique was administered in $13(65 \%)$ patients and $7(35 \%)$ patients received intensity modulated radiation therapy (IMRT). The median radiation dose in equivalent dose in 2 Gy fractions (EQD2) was 42 (range=5-70) Gy. Seven potential patient- and treatment-related features, namely age, gender, Karnofsky performance status (KPS), number of involved metastatic sites, surgery, concurrent and sequential chemotherapy and radiation dose escalation were analysed for their impact on OS and progression-free survival (PFS). Treatment-related side-effects were evaluated using Common Terminology Criteria for Adverse events (CTCAE) version 4.

Statistical analysis. Statistical analyses were performed using SPSS statistics 25 (IBM, New York City, NY, SA). The log-rank test was used to compare subgroups. All significant variables in univariate analysis were included in a multivariate analysis (Cox regression). OS was defined as the time between diagnosis and death. PFS was defined as the time between diagnosis and the development of local relapse, distant metastases or death from all causes.

For all statistical analyses, a $p$-value $<0.05$ was considered statistically significant.

\section{Results}

Median survival time was 2 (range=1-22) months. Survival at 1,3 and 12 months of the entire cohort was 65, 30 and $5 \%$, respectively. No local progression was observed in the first 12 months after radiotherapy. Progression-free survival was 2 (range $=1-20)$ months. In univariate analyses, surgery $(p=0.005)$, sequential or concurrent chemotherapy $(p=0.018)$ and radiation dose escalation ( $>39 \mathrm{~Gy}, p=0.038$ ) resulted in improved OS (Table II), respectively. Karnofsky performance status $>70 \%$ showed a trend for longer survival time $(p=0.062)$. On multivariate analysis, no factor achieved significance for OS. Limited metastatic sites (1 vs. 2-4 sites, $p=0.043)$, surgery $(p=0.024)$ and chemotherapy $(p=0.039)$ are associated with improved PFS (Table III). On multivariate analysis for PFS no factor achieved significance.

Acute toxicity (grade 3 ) of dysphagia, dyspnea, dermatitis, mucositis and dysphonia were found in $20 \%, 30 \%, 5 \%, 5 \%$ and $10 \%$ of patients, respectively. CTCAE grade $4 / 5$ was not observed.

\section{Discussion}

The treatment of patients with metastatic ATC should be planned in a multidisciplinary expert team also considering prognostic factors, which allow estimating the patient's prognosis $(1,10,12)$. Systemic treatment remains the main treatment of metastatic ATC, but usually results in low response rates and significant toxicities. Treatment with paclitaxel or doxorubicin or combined treatment approaches (e.g. carboplatin/paclitaxel, docetaxel/doxorubicin) are recommended (5).
Table I. Patient- and treatment-related characteristics.

\begin{tabular}{|c|c|c|}
\hline & $\mathrm{N}$ & $\%$ \\
\hline \multicolumn{3}{|l|}{ Age, years } \\
\hline$<73$ & 10 & 50 \\
\hline$\geq 73$ & 10 & 50 \\
\hline \multicolumn{3}{|l|}{ Gender } \\
\hline Female & 11 & 55 \\
\hline Male & 9 & 45 \\
\hline \multicolumn{3}{|c|}{ Karnofsky performance status, $\%$} \\
\hline$\leq 70$ & 14 & 70 \\
\hline$>70$ & 6 & 30 \\
\hline \multicolumn{3}{|l|}{$\mathrm{T}$ stage } \\
\hline 3 & 1 & 5 \\
\hline 4 & 19 & 95 \\
\hline \multicolumn{3}{|l|}{$\mathrm{N}$ category } \\
\hline 0 & 5 & 25 \\
\hline 1 & 15 & 75 \\
\hline \multicolumn{3}{|c|}{ Number of metastatic sites } \\
\hline 1 & 11 & 55 \\
\hline $2-4$ & 9 & 45 \\
\hline \multicolumn{3}{|l|}{ Metastatic sites } \\
\hline Pulmonary & 19 & 95 \\
\hline Distant lymph node & 8 & 40 \\
\hline Bone & 3 & 15 \\
\hline Brain & 1 & 5 \\
\hline Liver & 1 & 5 \\
\hline \multicolumn{3}{|l|}{ Surgery } \\
\hline No & 15 & 75 \\
\hline Yes & 5 & 25 \\
\hline \multicolumn{3}{|l|}{ Chemotherapy } \\
\hline No & 11 & 55 \\
\hline Yes & 9 & 45 \\
\hline \multicolumn{3}{|l|}{ Radiation dose, Gy } \\
\hline$\leq 39$ & 8 & 40 \\
\hline$>39$ & 12 & 60 \\
\hline
\end{tabular}

T: Tumor, N: nodal, Gy: Gray.

Tumor-related complications such as airway or esophageal obstruction, hemorrhage and vocal cord paralysis can be lethal and should be taken into account for shared-decision making (2-5). Therefore, prognostic factors that indicate the effect of radiotherapy on local control and survival are important to identify high-risk patients who do not achieve a satisfying outcome and may benefit from treatment intensification such as radiation dose escalation or concurrent chemotherapy.

Several studies investigated potential prognostic factors in ATC such as age, presence of acute symptoms, leukocytosis, large local tumors, resection status and distant metastasis (1, $13,14)$. Data about prognostic factors in stage IVC are still limited $(8,13,15)$.

The present study aimed to evaluate outcome and prognostic factors in patients with metastatic anaplastic thyroid cancer that received radiation to the primary tumor. As a result, cervical irradiation appears to be effective and offers durable local 
Table II. Uni- and multivariate analyses of overall survival.

\begin{tabular}{|c|c|c|c|c|}
\hline At 1 month, $\%$ & At 3 months, $\%$ & At 12 months, $\%$ & $\begin{array}{c}p \text {-Value } \\
\text { (univariate analyses) }\end{array}$ & $\begin{array}{c}p \text {-Value } \\
\text { (multivariate analyses) }\end{array}$ \\
\hline
\end{tabular}

\begin{tabular}{|c|c|c|c|c|c|}
\hline \multicolumn{6}{|l|}{ Age, years } \\
\hline$<73$ & 60 & 30 & 10 & & \\
\hline$\geq 73$ & 70 & 30 & 0 & 0.47 & \\
\hline \multicolumn{6}{|l|}{ Gender } \\
\hline Female & 64 & 27 & 9 & & \\
\hline Male & 67 & 33 & 0 & 0.802 & \\
\hline \multicolumn{6}{|c|}{ Karnofsky performance status, $\%$} \\
\hline$\leq 70$ & 57 & 21 & 0 & & \\
\hline$>70$ & 83 & 50 & 17 & 0.062 & \\
\hline \multicolumn{6}{|c|}{ Metastatic sites } \\
\hline 1 & 73 & 36 & 9 & & \\
\hline $2-4$ & 56 & 22 & 0 & 0.322 & \\
\hline \multicolumn{6}{|l|}{ Surgery } \\
\hline No & 53 & 13 & 0 & & \\
\hline Yes & 100 & 80 & 20 & 0.005 & 0.051 \\
\hline \multicolumn{6}{|c|}{ Chemotherapy } \\
\hline No & 44 & 11 & 0 & & \\
\hline Yes & 82 & 46 & 9 & 0.018 & 0.988 \\
\hline \multicolumn{6}{|c|}{ Radiation dose, Gy } \\
\hline$\leq 39$ & 38 & 13 & 0 & & \\
\hline$>39$ & 83 & 42 & 8 & 0.038 & 0.415 \\
\hline
\end{tabular}

Gy: Gray.

Table III. Uni- and multivariate analyses of progression-free survival.

At 1 Month, $\% \quad$ At 3 months, $\% \quad$ At 12 months, $\% \quad p$-Value $\quad p$-Value

(univariate analyses) (multivariate analyses)

\begin{tabular}{|c|c|c|c|c|c|}
\hline \multicolumn{6}{|l|}{ Age, years } \\
\hline$<73$ & 50 & 20 & 10 & \multicolumn{2}{|l|}{0.926} \\
\hline$\geq 73$ & 70 & 30 & 0 & & \\
\hline \multicolumn{6}{|l|}{ Gender } \\
\hline Female & 64 & 18 & 9 & \multirow{2}{*}{\multicolumn{2}{|c|}{0.896}} \\
\hline Male & 56 & 22 & 0 & & \\
\hline \multicolumn{6}{|c|}{ Karnofsky performance status, $\%$} \\
\hline$\leq 70$ & 50 & 14 & 0 & \multirow{2}{*}{\multicolumn{2}{|c|}{0.192}} \\
\hline$>70$ & 83 & 33 & 17 & & \\
\hline \multicolumn{6}{|l|}{$\mathrm{N}$ stage } \\
\hline 0 & 60 & 40 & 20 & \multirow{2}{*}{\multicolumn{2}{|c|}{0.263}} \\
\hline 1 & 60 & 13 & 0 & & \\
\hline \multicolumn{6}{|c|}{ Metastatic sites } \\
\hline 1 & 64 & 36 & 9 & \multirow{3}{*}{0.043} & \multirow[b]{2}{*}{0.336} \\
\hline $2-4$ & 56 & 0 & 0 & & \\
\hline \multicolumn{5}{|l|}{ Surgery } & \\
\hline No & 47 & 7 & 0 & \multirow[b]{2}{*}{0.024} & \multirow[b]{2}{*}{0.23} \\
\hline Yes & 100 & 60 & 20 & & \\
\hline \multicolumn{6}{|c|}{ Chemotherapy } \\
\hline No & 44 & 0 & 0 & \multirow{3}{*}{0.039} & \multirow[b]{2}{*}{0.598} \\
\hline Yes & 73 & 36 & 9 & & \\
\hline \multicolumn{5}{|c|}{ Radiation dose, Gy } & \\
\hline$\leq 39$ & 38 & 0 & 0 & \multirow{2}{*}{\multicolumn{2}{|c|}{0.075}} \\
\hline$>39$ & 75 & 33 & 8 & & \\
\hline
\end{tabular}

Gy: Gray. 
control of the disease. In our analysis surgery, chemotherapy (carboplatin/paclitaxel or doxorubicin) and radiation dose escalation (>39 Gy) were correlated with longer OS. This is consistent with the findings by Wendler et al. who reported a significant association of multimodal treatment approaches with a survival benefit in ATC stage IVC patients (1) as well as the study of Sugitani et al. with 223 ATC patients in stage IVC which found that radical surgery $(p=0.0002)$, dose escalated radiotherapy ( $\geq 40 \mathrm{~Gy})(p<0.0001)$ and chemotherapy $(p<0.0001)$ were correlated with longer survival rates (13).

A National Cancer Data Base (NCDB) study of 606 patients with ATC stage IVC found a dose-survival correlation in patients receiving 60 to 75 Gy compared to 45 to 59.9 Gy (15). These data go along with the findings of our present study.

Despite local and symptom control, pattern of failure in ATC tends to be metastatic progression, which is consistent with our study (16). We found that a limited number of metastatic sites ('oligometastatic disease') appears to be a prognostic factor for PFS (see Table III) and that these patients may benefit from more aggressive treatment approaches which offer reliable local control and longer OS.

Treatment-related side-effects were manageable according to our study. However, treatment intensification should be discussed in multidisciplinary teams including surgeons, radiation oncologists, endocrinologists, medical oncologists, and palliative care teams.

Due to a substantial increase of druggable tumor-specific molecular aberrations in the past decade, molecular profiling has become an integral part of diagnosis and therapy in oncology, gaining more and more importance in the treatment of ATC. The most frequently investigated single agents or agents in combined treatment approaches are multikinase inhibitors (e.g. lenvatinib, sorafenib), PI3K/mTOR inhibitors (e.g. everolismus), vascular-targeting agents (e.g. fosbretabulin), BRAF-inhibitors (e.g. dabrafenib) and checkpoint inhibitors (e.g. pembrolizumab, spartalizumab) (17-23).

The combination of BRAF-inhibitor dabrafenib and MEKinhibitor trametinib achieved approval by the Food and Drug Administration (FDA) in 2018 based on the promising results of a phase II study comprising BRAFV600E-mutated ATC (21). The overall response rate in the ATC-subgroup was $61 \%$, of which $57 \%$ experienced a partial and $4 \%$ a complete response. In addition, several checkpoint inhibitors such as pembrolizumab, spartalizumab and durvalumab alone or in combination with conventional treatment regimens (RT, ChT) have been investigated (22-24).

Recently, the combination of lenvatinib with pembrolizumab has achieved excellent results in metastatic ATC (25). However, larger trials investigating this combined treatment approach are ongoing and highly anticipated (26).

Several limitations must be considered in interpreting the results of our study such as the retrospective design and, therefore, a risk of including hidden selection biases. In addition, the patient cohort is relatively small with a long recruitment period. However, ATC is an orphan disease and stage IVC is associated with a dismal prognosis. We found that local irradiation offers durable local control in metastatic ATC patients and identified several prognostic factors in this setting. As a result, we are convinced that our study may help physicians to tailor personalized treatment approaches.

In summary, treatment-related side-effects appear to be manageable and therapy intensification including concurrent or sequential chemotherapy and radiation dose escalation were correlated with longer survival rates.

\section{Conclusion}

Radiation to the primary tumor in metastatic anaplastic thyroid cancer is safe and offers durable local control. Treatment intensification including concurrent or sequential chemotherapy and radiation dose escalation were correlated with longer survival rates and should be considered in selected patients with metastatic disease.

\section{Conflicts of Interest}

There are no conflicts of interest to declare regarding this study.

\section{Authors' Contributions}

Conception and Design: L.K., T.A., D.O.; Administrative support: C.B., C.S., L.K.; Provision of study materials or patients: L.K., C.S., C.B., J.R.; Collection and assembly of data: L.K., T.A., D.O.; Data analysis and interpretation: L.K., T.A., D.O.; Manuscript writing: All authors; Final approval of manuscript: All Authors.

\section{References}

1 Wendler J, Kroiss M, Gast K, Kreissl MC, Allelein S, Lichtenauer U, Blaser R, Spitzweg C, Fassnacht M and Schott M: Clinical presentation, treatment and outcome of anaplastic thyroid carcinoma: results of a multicenter study in Germany. Eur J Endocrinol 175(6): 521-529, 2016. PMID: 27926471. DOI: 10.1530/EJE-16-0574

2 Smallridge RC, Ain KB, Asa SL, Bible KC, Brierley JD, Burman KD, Kebebew E, Lee NY, Nikiforov YE and Rosenthal MS: American Thyroid Association guidelines for management of patients with anaplastic thyroid cancer. Thyroid 22(11): 11041139, 2012. PMID: 23130564. DOI: 10.1089/thy.2012.0302

3 Perros P, Boelaert K, Colley S, Evans C, Evans RM, Gerrard BA G, Gilbert J, Harrison B, Johnson SJ and Giles TE: Guidelines for the management of thyroid cancer. Clin Endocrinol (Oxf) 81(Suppl 1): 1-122, 2014. PMID: 24989897. DOI: 10.1111/cen.12515

4 Haddad R, Lydiatt W, Bischoff L, Busaidy N, Byrd D, Callender G, Dickson P, Duh Q, Ehya H and Haymart M: NCCN clinical practive guidelines in oncology (NCCN guidelines): thyroid carcinoma. Version 1, 2019. NCCN 2019. Available at: https:// www.nccn.org/professionals/physician_gls/pdf/thyroid.pdf [Last accessed July 1, 2019] 
5 Filetti S, Durante C, Hartl D, Leboulleux S, Locati L, Newbold K, Papotti M, Berruti A: Thyroid cancer: ESMO Clinical Practice Guidelines for diagnosis, treatment and follow-up. Ann Oncol 30(12): 1856-1883, 2019. PMID: 31549998. DOI: 10.1093/annonc/mdz400

6 Oliinyk D, Augustin T, Koehler VF, Rauch J, Belka C, Spitzweg $\mathrm{C}$ and Käsmann L: Hypofractionated radiotherapy for anaplastic thyroid cancer: Systematic review and pooled analysis. Cancers 12(9): 2506, 2020. PMID: 32899355. DOI: 10.3390/cancers 12092506

7 Brierley JD, Gospodarowicz MK and Wittekind C: TNM classification of malignant tumours. Chichester, West Sussex, UK Hoboken, Wiley Blackwell/John Wiley \& Sons, Inc, 2017.

8 Onoda N, Sugitani I, Ito K, Suzuki A, Higashiyama T, Fukumori $\mathrm{T}$, Suganuma N, Masudo $\mathrm{K}$, Nakayama $\mathrm{H}$ and Uno A: Evaluation of the 8th edition TNM classification for anaplastic thyroid carcinoma. Cancers 12(3): 552, 2020. PMID: 32120853. DOI: $10.3390 /$ cancers 12030552

9 Augustin T, Oliinyk D, Köhler VF, Rauch J, Belka C, Spitzweg C and Käsmann L: Clinical outcome and toxicity in the treatment of anaplastic thyroid cancer in elderly patients. J Clin Med 9(10): 3231, 2020. PMID: 33050286. DOI: 10.3390/jcm9103231

10 Käsmann L, Bolm L, Janssen S and Rades D: Prognostic factors for survival in patients treated with multimodal therapy for anaplastic thyroid cancer. Anticancer Res 36(9): 4697-4700, 2016. PMID: 27630315. DOI: 10.21873/anticancerres.11023

11 Fan D, Ma J, Bell AC, Groen AH, Olsen KS, Lok BH, Leeman JE, Anderson E, Riaz N and McBride S: Outcomes of multimodal therapy in a large series of patients with anaplastic thyroid cancer. Cancer 126(2): 444-452, 2020. PMID: 31593317. DOI: $10.1002 / \mathrm{cncr} .32548$

12 Rades D, Janssen S, Käsmann L, Bolm L and Schild SE: Outcomes after irradiation of epidural spinal cord compression due to metastatic thyroid cancer. Anticancer Res 36(4): 2035 2039, 2016. PMID: 27069199.

13 Sugitani I, Miyauchi A, Sugino K, Okamoto T, Yoshida A and Suzuki S: Prognostic factors and treatment outcomes for anaplastic thyroid carcinoma: ATC Research Consortium of Japan cohort study of 677 patients. World J Surg 36(6): 1247-1254, 2012. PMID: 22311136. DOI: 10.1007/s00268-012-1437-z

14 Käsmann L, Eze C and Manapov F: Stereotactic body radiation therapy (SBRT) combined with immune check-point inhibition (ICI) in advanced lung cancer: Which metastatic site should be irradiated to induce immunogenic cell death? Int J Radiat Oncol Biol Phys 108(1): 225-226, 2020. PMID: 32414625. DOI: 10.1016/j.ijrobp.2020.04.002

15 Pezzi TA, Mohamed AS, Sheu T, Blanchard P, Sandulache VC, Lai SY, Cabanillas ME, Williams MD, Pezzi CM and Lu C: Radiation therapy dose is associated with improved survival for unresected anaplastic thyroid carcinoma: outcomes from the National Cancer Data Base. Cancer 123(9): 1653-1661, 2017. PMID: 28026871. DOI: 10.1002/cncr.30493

16 Rao SN, Zafereo M, Dadu R, Busaidy NL, Hess K, Cote GJ, Williams MD, William WN, Sandulache V and Gross N: Patterns of treatment failure in anaplastic thyroid carcinoma. Thyroid 27(5): 672-681, 2017. PMID: 28068873. DOI: 10.1089/thy.2016.0395

17 Savvides P, Nagaiah G, Lavertu P, Fu P, Wright JJ, Chapman R, Wasman J, Dowlati A and Remick SC: Phase II trial of sorafenib in patients with advanced anaplastic carcinoma of the thyroid.
Thyroid 23(5): 600-604, 2013. PMID: 23113752. DOI: 10.1089/thy.2012.0103

18 Tahara M, Kiyota N, Yamazaki T, Chayahara N, Nakano K, Inagaki L, Toda $\mathrm{K}$, Enokida $\mathrm{T}$, Minami $\mathrm{H}$ and Imamura $\mathrm{Y}$ : Lenvatinib for anaplastic thyroid cancer. Front Oncol 7: 25, 2017. PMID: 28299283. DOI: 10.3389/fonc 2017.00025

19 Hanna GJ, Busaidy NL, Chau NG, Wirth LJ, Barletta JA, Calles A, Haddad RI, Kraft S, Cabanillas ME and Rabinowits G: Genomic correlates of response to everolimus in aggressive radioiodine-refractory thyroid cancer: a phase II study. Clin Cancer Res 24(7): 1546-1553, 2018. PMID: 29301825. DOI: 10.1158/1078-0432.CCR-17-2297

20 Mooney CJ, Nagaiah G, Fu P, Wasman JK, Cooney MM, Savvides PS, Bokar JA, Dowlati A, Wang D and Agarwala SS: A phase II trial of fosbretabulin in advanced anaplastic thyroid carcinoma and correlation of baseline serum-soluble intracellular adhesion molecule-1 with outcome. Thyroid 19(3): 233-240, 2019. PMID: 19265494. DOI: 10.1089/thy.2008.0321

21 Subbiah V, Kreitman RJ, Wainberg ZA, Cho JY, Schellens JH, Soria JC, Wen PY, Zielinski C, Cabanillas ME and Urbanowitz G: Dabrafenib and trametinib treatment in patients with locally advanced or metastatic BRAF V600-mutant anaplastic thyroid cancer. J Clin Oncol 36(1): 7, 2018. PMID: 29072975. DOI: 10.1200/JCO.2017.73.6785

22 Chintakuntlawar AV, Yin J, Foote RL, Kasperbauer JL, Rivera M, Asmus E, Garces NI, Janus JR, Liu M and Ma DJ: A phase 2 study of pembrolizumab combined with chemoradiotherapy as initial treatment for anaplastic thyroid cancer. Thyroid 29(11): 1615-1622, 2019. PMID: 31595822. DOI: 10.1089/thy.2019.0086

23 Sherman EJ, Tsai CJ, Zhi WI, Fetten JV, Wu V, Ho AL, Riaz N, Pfister DG and Lee NY: Pilot study combining PD-L1 antibody durvalumab (D) with CTLA-4 antibody tremelimumab (T) and stereotactic body radiotherapy (SBRT) to treat metastatic anaplastic thyroid cancer (ATC). J Clin Oncol 37(Suppl 15): 6088, 2019. DOI: 10.1200/JCO.2019.37.15_suppl.6088

24 Capdevila J, Wirth LJ, Ernst T, Ponce Aix S, Lin C-C, Ramlau R, Butler MO, Delord J-P, Gelderblom H, Ascierto PA, Fasolo A, Führer D, Hütter-Krönke ML, Forde PM, Wrona A, Santoro A, Sadow PM, Szpakowski S, Wu H, Bostel G, Faris J, Cameron S, Varga A and Taylor M: PD-1 blockade in anaplastic thyroid carcinoma. J Clin Oncol 38(23): 2620-2627, 2020. PMID: 32364844. DOI: $10.1200 / \mathrm{JCO} .19 .02727$

25 Dierks C, Miething C, Thomusch O, von Bubnoff N and Duyster $\mathrm{J}$ : Lenvatinib and pembrolizumab as save and effective combination treatment in 8 patients with metastasized anaplastic (ATC) or poorly differentiated thyroid carcinoma (PDTC). Ann Oncol 29(Suppl 8): VIII646, 2018. DOI: 10.1093/annonc/mdy302.004

26 Dierks C, Seufert J, Ruf J, Duyster J, Thomusch O and Miething C and Zielke A: 1915P The lenvatinib/pembrolizumab combination induces long lasting and complete responses in patients with metastatic anaplastic or poorly differentiated thyroid carcinoma: Results from a retrospective study and first results from the prospective phase II ATLEP trial. Ann Oncol 31(4): S1085, 2020. DOI: 10.1016/j.annonc.2020.08.1403

Received November 16, 2020

Revised November 29, 2020 Accepted December 1, 2020 